\title{
Study of Central-Line Associated Blood Stream Infections (CLABSIs) and Central-Line Related Blood Stream Infections (CRBSIs) in a Tertiary Hospital, Bangalore, India
}

\author{
C. Bhavana*, T. Nagarathnamma and R. Ambica
}

\author{
Department of Microbiology, Bangalore Medical College and Research Institute, \\ Karnataka, India \\ *Corresponding author
}

\section{A B S T R A C T}

\begin{tabular}{|l|}
\hline Ke y w or d s \\
Central line- \\
associated \\
bloodstream \\
infections, Central \\
line-related \\
bloodstream \\
infections, \\
Surveillance
\end{tabular}

Bloodstream infections caused by central venous catheter remains a serious and the most common cause of Hospital acquired infections (HAIs) worldwide. Central line-associated bloodstream infections remain a leading cause of serious healthcare-associated infections in ICUs in India, the rate being 7.9 per 1000 central line-days. Accurate measurement of the rates of BSIs arising from catheters is important because ICUs with high rates will need to institute further performance improvement initiatives in CVC insertion and management. The main aims of this study were to identify the various factors influencing the infections associated with CVC, the rate of infections associated with CVC, to identify the organisms involved in the causation of Central-line associated blood stream infections (CLABSIs) and Central-line related blood stream infections (CRBSIs) and to study the antibiotic susceptibility patterns of the isolated organisms. The present study revealed that rate of CLABSI was 8.26/1000 central-line days and CRBSI was $0.26 / 1000$ central-line days mostly affecting the age group 51-60 years and males were more commonly than females. Staphylococci were the most common organism isolated. The study showed high incidence of resistance against conventional antibiotics such as Ampicillin, Amoxycillin-clavulanate, Gentamicin, Amikacin, Erythromycin and Azithromycin among the pathogens causing CLABSI/CRBSI. Since CVCs are increasingly being used in the critical care, regular surveillance for infections associated with them is essential. There is a change in the pattern on pathogens causing the bloodstream infections and their susceptibility pattern as compared to other studies. Hence, it is very important for strengthening of the infection control, instituting surveillance systems, and implementing evidence-based preventive strategies in order to prevent bloodstream infections caused by CVCs.

\section{Introduction}

Bloodstream infections caused by central venous catheter remains a serious and the most common cause of Hospital acquired infections (HAIs) worldwide (Edwards et al., 2007). Central line-associated bloodstream infections remain a leading cause of serious healthcare-associated infections in ICUs in
India, the rate being 7.9 per 1000 central linedays (Mehta et al., 2007).

Central venous catheters (CVCs) are indispensable in modern-day medical practice, particularly in intensive care units (ICUs). They provide secure access to the central circulation for infusion therapy, nutritional support, hemodynamic monitoring, plasma 
pheresis, apheresis and hemodialysis. CVCs have a higher infection risk than other indwelling vascular access lines. This causes significant morbidity and mortality to the critically ill patient (Raad et al., 2007; Frasca et al., 2010). Although such catheters provide necessary vascular access, their use puts patients at risk for local and systemic infections, including local site infection, catheter related blood stream infections, septic thrombophlebitis, endocarditis, and other metastatic infections (e.g., lung abscess, brain abscess, osteomyelitis and endophthalmitis) (Khanna et al., 2013).

The CDC (Centers for Disease Control and prevention) defines a Central-line associated Blood Stream Infections (CLABSI) as a blood stream infection caused by an organism not related to another infection when a central line has been in place at some time during the 48 hr prior to the collection of the blood.

In contrast, a Central line-related Blood Stream Infections (CRBSI) is defined as a blood stream infection with either a positive catheter tip culture or a positive blood culture drawn from the central venous catheter consistent with a culture drawn simultaneously from a peripheral site (Horan et al., 2008).

Incidence of bloodstream infections in patients with indwelling catheter is directly related to factors such as site of catheterization, type of procedure, number of attempts, length of catheter inside, duration of catheterization, systemic antibiotics, local site infection of catheter, reason for catheter removal, experience of venipuncturist (Patil et al., 2011).

Patients with abrupt onset of signs and symptoms of sepsis without any identifiable source should prompt suspicion of infection of an intravascular device (Brachman, 2007).
The incidence of BSIs associated with peripheral venous catheters is usually low even though they are used most frequently for vascular access. The majority of serious catheter-related infections are associated with central venous catheters (CVCs), especially those that are placed in patients in ICUs (Naomi et al., 2002).

Vascular catheters have become an increasingly important source of bacteremia, increasing from $3 \%$ in the mid-1970s to $19 \%$ in the early 1990s.

Primary bacteremia, including intravascular catheter sources, account for approximately one half of all ICU-related bacteremia (Beekmann and Henderson, 2015). A total of 250,000 cases of CVC-associated BSIs have been estimated to occur annually if entire hospitals are assessed rather than ICUs exclusively.

Attributable mortality is an estimated $12 \%$ $25 \%$ for each infection, and the marginal cost to the health-care system is $\$ 25,000$ per episode (Kluger and Maki, 1999).

Staphylococci continue to predominate as the most frequently encountered pathogens in device-related infections. Other commonly encountered isolates include Enterococcus spp. Serratia marcescens, Candida albicans, Candida tropicalis, Pseudomonas aeruginosa, Klebsiella spp., Enterobacter spp., Citrobacter freundii, Burkholderia cepacia complex, Acinetobacter baumannii (Beekmann and Henderson, 2015).

Accurate measurement of the rates of BSIs arising from catheters is important because ICUs with high rates of CRBSIs will need to institute further performance improvement initiatives in CVC insertion and management. By contrast, ICUs that have high rates of CLABSIs will find that changes in CVC-based 
interventions are ineffective (Sihler et al., 2010). This study was undertaken among patients admitted in PMSSY (Pradhan Mantri Swasthya Suraksha Yojana - Super speciality departments including Paediatric surgery, Surgical Gastroenterology, Neurosurgery, Neurology, Cardiology), Victoria hospital, Bowring and Lady Curzon Hospital, Vani Vilas Hospital attached to Bangalore Medical College and Research Institute to determine the rate of bloodstream infections caused by CVC and to identify the factors influencing it, which would help to institute better prophylactic measures.

\section{Materials and Methods}

The present prospective study was undertaken in the Department of Microbiology, Bangalore Medical College and Research Institute, Bangalore from November 2014 - October 2016.

The study included 150 patients admitted in Intensive care units in PMSSY, Victoria hospital, Bowring and Lady Curzon Hospital, Vani Vilas Hospital attached to Bangalore Medical College and Research Institute with central venous catheter in place for the past 48 hrs or more.

\section{Aims and objectives}

To study the various factors influencing the infections associated with CVC.

To study the rate of infections associated with CVC.

To identify the organisms involved in the causation of Central-line associated blood stream infections (CLABSIs) and Central-line related blood stream infections (CRBSIs).

To study the antibiotic susceptibility patterns of the isolated organisms.

\section{Inclusion criteria}

Patients presenting with clinical symptoms and signs of bacteraemia when the central venous catheter was in place for the past $48 \mathrm{hrs}$ and meet the criteria according to CDC definitions of CLABSI and CRBSI as follows:

Centres for Disease Control and Prevention Definitions of Catheter-Associated and Catheter-Related Blood Stream Infection (Horan et al., 2008).

Catheter-Associated blood stream infections -

Criterion 1: Patient has a recognized pathogen cultured from 1 or more blood cultures and organism cultured from blood is not related to an infection at another site.

Criterion 2: Patient has at least one of the following signs or symptoms: fever $\left(>38^{\circ} \mathrm{C}\right)$, chills, or hypotension and signs and symptoms and positive laboratory results are not related to an infection at another site and at least one of the following:

Common skin contaminant (e.g., diphtheroids, Bacillus spp., Propioni bacterium spp., coagulase-negative Staphylococci or Micrococci) is cultured from two or more blood samples drawn on separate occasions.

Common skin contaminant is cultured from at least one blood culture from a patient with an intravascular catheter, and the physician institutes appropriate antimicrobial therapy.

Catheter-Related blood stream infection is considered if positive blood culture with the same microorganism present in the tip of the catheter.

Quantitatively or semi-quantitatively evaluated and clinical and microbiological absence of another focus of infection. 


\section{Exclusion criteria}

Patients with obvious source of infection (fever, pneumonia, urinary tract infection and cellulitis) other than central venous catheter by history, clinical examination, blood culture, chest X-ray, urine examination, etc. and relevant investigations pertaining to the suspected infection were excluded.

\section{History taking}

Detailed history of each case was taken regarding name, age, sex, ward, diagnosis, purpose of catheterization, site of catheter, duration of catheterization, length of catheter in situ, presenting symptoms leading to removal of catheter, type of procedure, number of attempts, experience of venipuncturist and any history of systemic antibiotics.

Sample collection and processing (Maki et al., 1977; Isenberg, 2007)

\section{Catheter tip collection}

The skin was cleaned with $70 \%$ alcohol prior to catheter removal. The catheter was held at the proximal end and carefully removed from the patient with a sterile instrument, taking care to avoid contact with the skin. The distal $5 \mathrm{~cm}$ was cut with sterile blade and collected in a sterile tube and transported to the lab as soon as possible.

\section{Catheter tip processing}

Extraluminal Maki's roll over plate method and endoluminal catheter flush culture was used for processing. It was performed on MacConkey agar and Blood agar.

\section{Extraluminal Maki's roll over method}

Forceps was dipped in 95\% alcohol, flame sterilized and allowed to cool. The catheter tip was transferred from transport container to agar plate using sterile forceps. The catheter tip was rolled back and forth across agar surface using slight pressure at least four times. It was made sure that the catheter tip was having good contact with the surface of the plate. The plates were incubated for at least $72 \mathrm{hrs}$ at $35^{\circ} \mathrm{C}$ in a $\mathrm{CO}_{2}$ incubator.

\section{Endoluminal catheter flush culture}

The catheter segment was aseptically transferred to a sterile test tube ( 12 by 75 $\mathrm{mm}$ ). Using a sterile syringe $1 \mathrm{ml}$ of sterile trypticase soya broth was drawn. A needle was placed into lumen of catheter and the broth was dispensed through catheter segment. The tube was capped and vortexed to dislodge adherent bacteria. The sample was serially diluted 100-folds. $100 \mu \mathrm{l}$ of each dilution was inoculated onto agar plates. Cross-streaks were made for well isolated colonies. The plates were incubated for $72 \mathrm{hrs}$ at $35^{\circ} \mathrm{C}$ in a $\mathrm{CO}_{2}$ incubator.

\section{Collection of peripheral blood sample and processing}

Blood sample collected under aseptic precautions from the peripheral vein in Brain Heart Infusion broth for qualitative culture were incubated at $37^{\circ} \mathrm{C}$. Subcultures were made on MacConkey's and blood agar plates after 24, 48 and $72 \mathrm{hr}$ and incubated for $24 \mathrm{hrs}$ at $37^{\circ} \mathrm{C}$.

\section{Identification and interpretation}

Catheter tip culture: Agar plates were examined at 24, 48 and 72 hrs. Significant growth is defined as $\geq 15$ colony forming units (CFU) by Maki's roll over plate method or $\geq$ $10^{3} \mathrm{CFU} / \mathrm{ml}$ by the endoluminal catheter flush method.

Blood culture: Agar plates were examined at 24, 48 and 72 hrs. 
The organisms were identified by colony morphology, gram staining, and biochemical tests performed by routine laboratory techniques (Koneman et al., 1997).

\section{Antibiotic sensitivity testing}

Antibiotic sensitivity pattern was interpreted using Kirby-Bauers disc diffusion method as recommended by Clinical Laboratory Standard Institute (CLSI) (Clinical and Laboratory Standards Institute, 2014).

\section{Results and Discussion}

Out of 150 cases studied, $95(63.33 \%)$ were male and $55(36.67 \%)$ were female. Male to female ratio was $1.73: 1$. The mean age for males and females was 43.48 years and 42.87 years respectively. The most common age group studied was 51-60 years of which 70\% and $30 \%$ were males and females respectively. This was followed by 1-20 years (16\%; $54.16 \%$ males and $45.84 \%$ females), $31-40$ years $(14 \%$; $66.67 \%$ males and $33.33 \%$ females) and $41-50$ years (14\%; $71.43 \%$ and $28.57 \%$ ).

In our study most common indication for central line insertion was hemodialysis (58.67\%), followed by infusion of intravenous fluids, medications and hemodynamic monitoring (40\%). Majority of the study group was distributed in Nephrology department (58.67\%), followed by Medicine (8.67\%), Paediatric surgery $(7.33 \%)$ and remaining $25.32 \%$ distributed among other departments.

Site of catheterization, type of procedure, no. of attempts, duration of catheter in situ and experience of venipuncturist were found to be significant factors. CLABSI rate was found to be 8.26/1000 central-line days and CRBSI was $0.14 / 1000$ central-line days, the total no. of central line days being 1452 during the study period. CRLI (Central line related local infections)/colonization was seen in $12.67 \%$ of the cases, in which only central line tip was culture positive with no evidence of bloodstream infections (Table 1). Catheter colonization did not appear to have direct bearing on blood stream infection.

Out of 95 samples studied among male patients, the no. of CLABSIs were 9 i.e. $9.47 \%$ of the males were affected. And, out of 55 samples studies among female patients, the no. of CLABSIs were found to be 3 i.e. 5.45\% of the females were affected. Whereas, both the cases of CRBSIs were found to be among male patients.

Overall, males constituted to (11/14) $78.57 \%$ and females (3/14) $21.43 \%$ of CLABSI and CRBSI cases.

The percentage of CLABSI was highest in the age groups 51-60 yrs (13.33\%), 1-20 yrs (12.5\%), 61-70 yrs (10.52\%) followed by <1 yr (5.56\%), 31-40 yrs and 41-50 yrs each being $4.76 \%$. Both the cases of CRBSI were found among the age group 51-60 yrs. Overall, the age group 51-60 yrs had highest incidence (5/14) of CVC associated and related blood stream infections which was found to be $35.71 \%$.

Blood stream infection was statistically significant among patients with IJV, emergency procedure, no. of attempts being 2 , duration of catheter in situ more than or equal to 6 days and experience of the venipuncturist with $\mathrm{p}$-value $<0.05$.

As the p-value for the factors- use of systemic antibiotics, presence of local site infection was $>0.05$, the development of CLABSI/CRBSI was not dependent on these 2 factors in the present study. The association with the length of catheter in situ could not be correlated as all the patients in the present study had length of the catheter in situ $<20 \mathrm{~cm}$. 
We found out that in our study, there was increased odds of developing CLABSI/CRBSI in the patients in whom the site of CVC insertion was IJV, emergency procedure, no. of attempts being more than 2, duration of catheter in situ more than or equal to 6 days and catheter being inserted by inexperienced venipuncturist.

The frequency of developing blood stream infection was found to be highest for the route of insertion being IJV, followed by femoral vein and least for subclavian vein.

In the present study, majority of CLABSI cases were bacterial which was 10/12 $(83.33 \%)$ remaining $2 / 12 \quad(16.67 \%)$ being fungal. Whereas, among 2 cases of CRBSI, 1 was bacterial and 1 was fungal growth.

In the present study, out of 10 bacterial cases in CLABSI, 6 (60\%) were caused by Gram positive isolates and remaining $4(40 \%)$ by Gram negative isolates.

In CRBSI, 1 bacterial case isolated was caused by Gram positive organism.

In the present study, Staphylococcus aureus $(42.86 \%)$ was the most common organism isolated, out of which MSSA accounted to (28.57\%) and MRSA (14.29\%), followed by Candida tropicalis (21.44\%).

Acinetobacter baumannii, Pseudomonas aeruginosa, Escherichia coli, Klebsiella pneumonia and Enterococcus fecalis were isolated in $7.14 \%$ cases each.

MSSA represented $4(28.57 \%)$ among 14 isolates, out of which all were sensitive $(100 \%)$ to Vancomycin, Cotrimoxazole, Linezolid, Tetracycline, Doxycycline and Ciprofloxacin. All the isolates were resistant to Penicillin G. Sensitivity to the rest of the drugs is as follows i.e., $3(75 \%)$ sensitive to
Clindamycin and Chloramphenicol, 2 (50\%) to Erythromycin, Azithromycin and Gentamicin.

Out of this, MSSA was isolated in a case of CRBSI, the sensitivity pattern being same in both tip culture and blood culture.

MRSA represented $2(12.5 \%)$ among 14 isolates, out of which both were sensitive (100\%) to Vancomycin, Linezolid, Tetracycline and Cotrimoxazole. Both the isolates were resistant to Penicillin G. 1 isolate (50\%) was sensitive to Doxycycline.

Candida tropicalis accounted to $3(21.44 \%)$ of the total 14 organisms isolated, in which 2 were isolated in CLABSI and 1 accounted for a case of CRBSI.

All the Candida tropicalis strains were susceptible to fluconazole (100\%).

E. coli and $K$. pneumonia were isolated in 1 each $(7.14 \%)$ of the 14 organisms. Both the isolates were sensitive (100\%) to Piperacillintazobactum, Cefepime, Ciprofloxacin and Imipenem. Both the isolates were resistant to Amoxycillin-clavulanate. Further, E. coli was sensitive to Gentamicin, Amikacin, Cefoxitin and Cefotaxime. K.pneumoniaewas sensitive to Cotrimoxazole.

$P$. aeruginosa and $A$. baumannii were isolated in 1 each $(7.14 \%)$ of the 14 organisms. $P$. aeruginosa was sensitive to Ciprofloxacin, Polymyxin $\mathrm{B}$ and Colistin, whereas $A$. baumannii was resistant to all the routine and reserved drugs.

Enterococcus faecalis represented 1 (7.14\%) among 14 isolates, which was sensitive (100\%) to Ampicillin, Penicillin, Vancomycin and Linezolid. It was resistant to High level Gentamicin. 
Table.1 Blood culture and CVC tip culture results

\begin{tabular}{|c|l|c|c|c|}
\hline $\begin{array}{c}\text { Blood } \\
\text { culture }\end{array}$ & $\begin{array}{c}\text { Tip } \\
\text { culture }\end{array}$ & Impression & No. of cases & $\begin{array}{c}\text { Rate per 1000 } \\
\text { central line days }\end{array}$ \\
\hline Positive & Negative & CLABSI & $12(8 \%)$ & 8.26 \\
\hline Positive & Positive & CRBSI & $2(1.33 \%)$ & 0.14 \\
\hline Negative & Positive & Catheter Tip Colonization & $19(12.67 \%)$ & \\
\hline Negative & Negative & No CLABSI/CRBSI & $117(78 \%)$ & \\
\hline
\end{tabular}

Table.2 Comparison of organisms isolated

\begin{tabular}{|l|l|l|l|l|l|l|}
\hline Study & $\begin{array}{l}\text { GahlotR } \\
\text { et al., } \\
(2013)\end{array}$ & $\begin{array}{l}\text { Datta et al., } \\
(\mathbf{2 0 1 4 )}\end{array}$ & $\begin{array}{l}\text { Cheewinmethasiri } \\
\text { et al., (2014) }\end{array}$ & $\begin{array}{l}\text { Kaur et } \\
\text { al., }\end{array}$ & $\begin{array}{l}\text { Mittal } \\
\text { G } \text { et al., } \\
(\mathbf{2 0 1 6})\end{array}$ & $\begin{array}{l}\text { Present } \\
\text { study }\end{array}$ \\
\hline S.aureus & $71 \%$ & $12.36 \%$ & $21.45 \%$ & $27.6 \%$ & $63 \%$ & $42.86 \%$ \\
\hline Candida spp & $7 \%$ & $4.5 \%$ & $21.45 \%$ & $17.2 \%$ & - & $21.44 \%$ \\
\hline A.baumannii & $22 \%$ & $26.97 \%$ & $6 \%$ & $10.3 \%$ & $15 \%$ & $7.14 \%$ \\
\hline P.aeruginosa & - & $14.61 \%$ & $8.3 \%$ & $20.6 \%$ & $9 \%$ & $7.14 \%$ \\
\hline E.coli & - & $2.25 \%$ & - & - & $3 \%$ & $7.14 \%$ \\
\hline K.pneumoniae & - & $29.2 \%$ & $12.75 \%$ & $10.3 \%$ & $9 \%$ & $7.14 \%$ \\
\hline E.faecalis & - & $10.11 \%$ & $3.75 \%$ & $14.8 \%$ & - & $7.14 \%$ \\
\hline CONS & - & - & $15.05 \%$ & - & - & - \\
\hline Enterobacterspp. & - & - & $10.55 \%$ & - & - & - \\
\hline
\end{tabular}

Culture plates showing growth from Maki's Roll over method

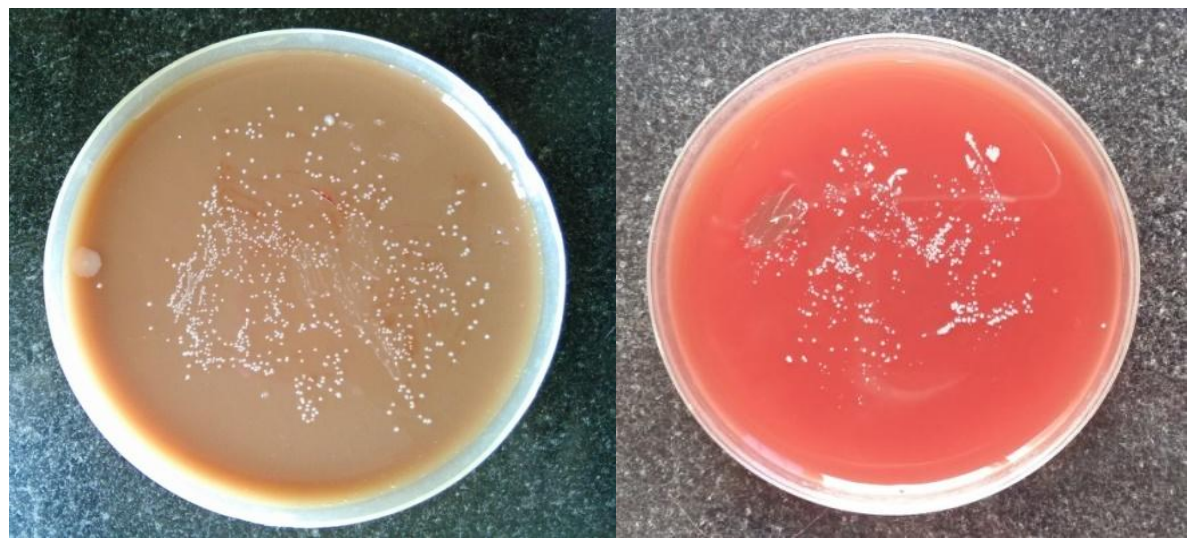

Note: Significant growth defined as $\geq 15$ colony forming units (CFU) is seen in the above pictures

Central venous catheters are the most frequently used indwelling medical devices and have become necessary tools for the successful treatment of patients with chronic or critical illness.
Our study investigated the rate, microbiological profile, antibiotic profile and the risk factors for the development of central line-associated infections in a tertiary care hospital. In the present study, the rate of 
CLABSI was 8.26/1000 central-line days in comparison with the study conducted by Mehta et al., (2007) (7.9), Deepthi et al., (2014) (8.2), Parameswaran et al., (2011) (8.75), El-Kholy et al., (2012) (9.1), Porto et al., (2010) (9.5) and Mittal et al., (2016) (9.5). Males $(78.57 \%)$ were more commonly affected than females $(21.43 \%)$. This correlates with the study of Khanna et al., (2013) (72.7\%), and other studies such as Apostolopoulou et al., (2009), Bicudo et al., (2011) and Datta et al., (2014) where the incidence of bloodstream infections were more in males than females.

The male gender has been stated as risk factor for the development of CLABSI/CRBSI in most of the studies. However, the reason for this high incidence in males could not be attributable to any reasons. CLABSI/CRBSI were common in the age group 51-60 years, in comparison with the study conducted by Brito et al., (2007), Apostolopoulou et al., (2009) and Datta et al., (2014). This could be attributable to the decreased immune status at that age group.

In the present study, $9.33 \%$ samples were culture positive in comparison with the study of Patil et al., (2011) (7.41\%), El-Kholy et al., (2012) (8.2\%), Porto et al., (2010) (11.2\%) and Apostolopoulou et al., (2009) (11.8\%). Lorente et al., (2005) and Brito et al., (2007) reported culture positivity as $2.04 \%$ and $3.8 \%$ respectively which was lesser compared to the present study. The comparison of organisms isolated with other studies is shown in Table 2.

The most important predisposing factors for developing bloodstream infections were the site of CVC insertion being IJV, emergency procedure, no. of attempts being more than 2 , duration of catheter in situ more than or equal to 6 days and catheter being inserted by inexperienced venipuncturist.
Overall, in the present study, there were basic similarities in the organisms isolated as compared with other studies that Gram positive organisms were predominant compared to Gram negative organisms which mainly consisted of Staphylococcus and predominant Gram negative organisms isolated were Pseudomonas aeruginosa, Klebsiella pneumoniae, Escherichia coli and Acinetobacter baumannii as in other studies, even though there was differences in the percentage of organisms isolated which could be attributable to differences in prevalence of organisms in different geographical areas.

In the present study, the most effective antibiotics against Gram positive isolates were Vancomycin, Linezolid (100\% each), Cotrimoxazole, Tetracycline (85.7\% each), Doxycycline (71.4\%) and Ciprofloxacin $(57.14 \%)$. They were least sensitive to Erythromycin, Azithromycin, Gentamicin (33.33\%) and Penicillin (14.29\%).

The most effective antibiotics against Gram negative isolates were Ciprofloxacin (100\%), Piperacillin-tazobactum, Cefepime and Imipenem (66.67\% each). They were least sensitive to Gentamicin and Amikacin (33.33\% each). A. baumannii was resistant to all the drugs. Fluconazole was effective against all the fungal bloodstream infections in the present study.

From the study, it can be concluded that since central venous catheters are increasingly being used in the critical care, regular surveillance for infections associated with them is essential.

The data showed high incidence of resistance against conventional antibiotics such as Ampicillin, Amoxycillin-clavulanate, Gentamicin, Amikacin, Erythromycin and Azithromycin among the pathogens causing CLABSI/CRBSI. 
Preventive measures against these infections include placement and maintenance of these catheters by skilled medical team, coating of catheters with antiseptic agents, topical disinfectants such as chlorhexidine, chlorhexidine-impregnated sponge dressing. The importance of strict asepsis has to be reinforced. All the intensivists are required to follow standard protocols that uniformly demand the use of sterile gowns, gloves, masks and large drapes during insertion to reduce $\mathrm{CVC}$ associated infection.

There is a change in the pattern on pathogens causing the bloodstream infections and their susceptibility pattern as compared to other studies which could be attributable to different geographical areas. Hence, it is very important for strengthening of the infection control, instituting surveillance systems, and implementing evidence-based preventive strategies in order to prevent bloodstream infections caused by central venous catheters.

\section{Limitations of the study}

The rate of blood stream infections in the present study could be affected by the small sample size of the study, less central-line days in our institution and the multitude of antibiotics received before the development of infection.

\section{Acknowledgement}

We thank the institute Bangalore Medical College and Research Institute to conduct this study.

\section{References}

Apostolopoulou E, Raftopoulos V, Filntisis G. Surveillance of device-associated infection rates and mortality in 3 Greek intensive care units. Am J Infect Control. May 2009; 22(3): 12-20.
Beekmann SE, and Henderson DK. Infections caused by percutaneous intravascular devices. In: Bennett JE, Mandell RD, editors. Mandell, Douglas and Bennett's principles and practice of infectious diseases, $8^{\text {th }}$ edition, Philadelphia: Elsevier; 2015. Pp. 3310-3324.

Bicudo D, Batista R, Henrique G, Furtado, Sola A, Alexandrino E. Risk factors for catheter-related bloodstream infection: a prospective multicenter study in Brazilian intensive care units. Braz J Infect Dis. 2011; 15(4): 328-331.

Brachman PS. The intensive care unit. In: Bennett JV, Brachmann PS, editors. Hospital Infections. $\quad 5^{\text {th }}$ edition. Philadelphia: Lippincott Williams and Wilkins; 2007. p. 395-416.

Brito CS, Gondim BA, Filho AD, Filho PG. Etiology and pathogenesis of bloodstream infections associated with the use of long-term central vascular catheter (CVC) in patients who undergone gastrointestinal surgery. Braz J Infect Dis. 2007; 11(1): 96-99.

Cheewinmethasiri J, Chittawatanarat K, Chandacham K, Jirapongchareonlap T, Chotirosniramit N. Microbiology, risk factors and mortality of patients with intravenous catheter related blood stream infections in the surgical intensive care unit: a five-year, concurrent, case-controlled study. J Med Assoc Thai. 2014 Jan; 97(1): S93101.

Clinical and Laboratory Standards Institute. Performance Standards for Antimicrobial Susceptibility Testing. Twenty-Fourth Informational Supplement. M100-S24, Vol 34, No 1: Wayne, Pennsylvania; 2014. p. 38-42.

Datta P, Rani H, Chauhan R, Gombar S, Chander J. Health-care-associated infections: Risk factors and epidemiology from an intensive care 
unit in Northern India. Indian J Anaesth. 2014 Jan-Feb; 58(1): 30-35.

Deepti, Sinha S, Sharma KS, Agarwal P, Biswas A, Sood S. Central Venous Catheter Related Bloodstream Infections in Medical Intensive Care Unit Patients in a Tertiary Referral Centre. Indian J Chest Dis Allied Sci 2014; 56: 85-91.

Edwards JR, Peterson KD, Andrus ML, Tolson JS, Goulding JS, Dudeck MA. National Healthcare Safety Network (NHSN) Report, data summary for 2006. Am J Infect Control. 2007; 35:290-301.

El-Kholy A, Saied T, Gaber M, Younain MA, Haleim MM, El-Sayed. Deviceassociated nosocomial infection rates in intensive care units at Cairo University hospitals: First step toward initiating surveillance programs in a resourcelimited country. Am J Infect Control 2012; 40: e216-20.

Frasca D, Dahyot-Fizelier C, Mimoz O. Prevention of central venous catheterrelated infection in the intensive care unit. Crit Care. 2010; 14: 212.

Gahlot R, Nigam C, Kumar V, Gupta M. Catheter Related Blood Stream Infections in ICU: A study from North India. Int J Infect Control. 2013; 9(2): 1-4.

Horan TC, Andrus M, Dudeck MA. CDC/NHSN surveillance definition of healthcare-associated infection and criteria for specific types of infections in the acute care setting. Am J Infect Control. 2008; 36: 309-332.

Isenberg HD.Culture of intravascular devices. In: Isenberg HD, Lynne S. Garcia, editors. Clinical Microbiology Procedures Handbook, 2nd edition. Washington DC: ASM Press; 2007. Vol.3. Section.13.12; p. 1-6.

Kaur M, Gupta V, Gombar S, Chander J, Sahoo T. Incidence, risk factors, microbiology of venous catheter associated bloodstream infections - A prospective study from a tertiary care hospital. Indian J Med Microbiol. 2015; 33(2): 248-254.

Khanna V, Mukhopadhayay C, Vandana KE, Verma M, Dabke P. Evaluation of Central Venous Catheter Associated Blood Stream Infections: A Microbiological Observational Study. J Pathog. 2013; 2013: 1-6.

Kluger DM, and Maki DG. The relative risk of intravascular device related bloodstream infections in adults. In: Abstracts of the 39th Interscience Conference on Antimicrobial Agents and Chemotherapy. San Francisco, CA: American Society for Microbiology, 1999: 514.

Koneman EW, Allen SD, Janda WM, Schreckenberger PC, Winn WC Jr. Charts. In: Darcy $\mathrm{P}$, Pterson $\mathrm{N}$, Montalbano J, editors. Koneman's Color Atlas and Textbook of Diagnostic Microbiology. 6th edition. Philadelphia: Lippincott; 1997. p. 1442-1535.

Lorente L, Henry C, Martin M et al., Central venous catheter-related infection in a prospective and observational study of 2,595 catheters. Crit care. Jul 2005; 9: R631-R635.

Maki DG, Weise CE, Sarafin HW. A semiquantitative culture method for identifying intravenous catheter-related infection. N Engl J Med. 1977; 296: 1305-9.

Mehta A, Rosenthal VD, Mehta Y, et al., Device-associated nosocomial infection rates in intensive care units of seven Indian cities. Findings of the International Nosocomial Infection Control Consortium (INICC). J Hosp Infect. 2007 Oct; 67(2): 168-174.

Mittal G, Gaind R, Verma PK, Deb M. Central venous catheter-related bloodstream infections in an intensive 
care unit from a tertiary care teaching hospital in India. Int $\mathbf{J}$ Infect Control. 2016; 12(1): 1-6.

Naomi P, Alexander M, Dellinger EP, et al., Guidelines for the Prevention of Intravascular Catheter-Related Infections. Centers for Disease Control and prevention. 2002 August; 51: 1-26.

Parameswaran R, Jatan B, Varma M, Mukhopadhyay C, Vidyasagar S. Intravascular catheter-related infections in an Indian tertiary care hospital. J Infect DevCtries 2011; 5(6): 452-458.

Patil HV, Patil VC, Ramteerthkar MN, Kulkarni RD. Central venous catheterrelated bloodstream infections in the intensive care unit. Indian $\mathbf{J}$ Crit Care Med. 2011; 15(4):213-223.

Porto JP, Dantas RC, Freitas C, Matoso DC, Almeida AB, Filho PG. Bloodstream infection associated/related to the central venous catheter in mixed ICU of adults from a Brazilian university hospital: Etiology, pathogenesis and risk factors. Rev PanamInfectol 2010; 12(2): 24-29.

Raad I, Hanna H, Maki D. Intravascular catheter-related infections: advances in diagnosis, prevention, and management. Lancet Infect Dis. 2007; 7: 645-57.

Sihler KC, Chenoweth C, Zalewski C, Wahl W, Hyzy R, Napolitano LM. CatheterRelated vs. Catheter-Associated Blood Stream Infections in the Intensive Care Unit: Incidence, Microbiology, and Implications. Surg. Infections. 2010; 11(6): 529-534.

\section{How to cite this article:}

Bhavana, C., T. Nagarathnamma and Ambica, R. 2018. Study of Central-Line Associated Blood Stream Infections (CLABSIs) and Central-Line Related Blood Stream Infections (CRBSIs) in a Tertiary Hospital, Bangalore, India. Int.J.Curr.Microbiol.App.Sci. 7(05): 697707. doi: https://doi.org/10.20546/ijcmas.2018.705.084 